\title{
LITERATUR
}

\section{Das Experiment einer europäischen Verfassung}

\author{
Martin Große Hüttmann*
}

Nach der Ablehnung des Verfassungsvertrags in den Referenden in Frankreich und in den Niederlanden im Mai 2005 wurde dieses Dokument von einigen Europapolitikern und Kommentatoren für tot erklärt. Als dann noch die britische Regierung wenige Tage nach dem „Aufstand in Europa“1 ihr für 2006 geplantes Referendum auf Eis gelegt hatte, schienen die Krise der europäischen Integration komplett und alle Hoffnungen, die mit diesem Verfassungsvertrag verknüpft wurden, hinfällig geworden zu sein. ${ }^{2}$ Obwohl oder gerade weil die Europäische Union im Frühsommer 2005 offensichtlich in eine Krise geraten war, bleibt die Beschäftigung mit der europäischen Konstitutionalisierung auf der Tagesordnung. Die hier vorzustellenden Bücher verlieren angesichts der neuen und veränderten Situation nichts von ihrer Bedeutung ganz im Gegenteil. Denn in einer Situation der Unsicherheit und Aufgeregtheit können theoretisch wie empirisch überzeugende Analysen den eingetrübten Blick klären und den politisch Verantwortlichen Handlungsanleitung geben. Das Thema als solches hat in den letzten Jahren in der politik- wie auch der rechtswissenschaftlichen EU-Forschung eine wahre Flut von Beiträgen und Sammelbänden gebracht, von der hier nur eine kleine Auswahl vorgestellt werden kann. ${ }^{3}$

Alle hier zu diskutierenden Bände beschäftigen sich mit dem ,Verfassungswerdungsprozess', der in der Folge der beinahe gescheiter-
Peter Becker und Olaf Leiße: Die Zukunft Europas. Der Konvent zur Zukunft der Europäischen Union, VS Verlag für Sozialwissenschaften: Wiesbaden 2005, ISBN 3-531-14100-7; 301 Seiten, $26,90 €$.

Erhard Busek und Waldemar Hummer (Hrsg.): Der Europäische Konvent und sein Ergebnis. Eine Europäische Verfassung, Böhlau Verlag: Wien u.a. 2004, ISBN 3-205-77227-X; 379 Seiten, $55 €$.

Mathias Jopp und Saskia Matl (Hrsg.): Der Vertrag über eine Verfassung für Europa. Analysen zur Konstitutionalisierung der EU, Nomos Verlagsgesellschaft: Baden-Baden 2005, ISBN 38329-1430-7; 563 Seiten, $69 €$.

Ulrike Liebert, Josef Falke, Kathrin Packham und Daniel Allnoch (Hrsg.): Verfassungsexperiment. Europa auf dem Weg zu zur transnationalen Demokratie?, Lit Verlag: Münster 2003, ISBN 3-8258-7102-9; 335 Seiten, 19,90 €.

Wolfgang Mantl, Sonja Puntscher Riekmann und Michael Schweitzer (Hrsg.): Der Konvent zur Zukunft der Europäischen Union, Böhlau Verlag: Wien u.a. 2005, ISBN 3-205-77127-3; 185 Seiten, $35 €$.

Werner Weidenfeld (Hrsg.): Die Europäische Verfassung in der Analyse, Verlag Bertelsmann Stiftung, Gütersloh 2005, ISBN 3-89204-727-8; 301 Seiten, $40 €$.

ten Regierungskonferenz 2000 („Nizza“) an Fahrt aufgenommen und die bisherige, stille

* Dr. des Martin Große Hüttmann, Institut für Politikwissenschaft, Universität Tübingen.

1 Der Spiegel Nr. 23, 06.06.2005.

2 Vgl. „Chirac désavoué, l'Europe déstabilisée“, in: Le Monde, 31.5.2005 und „EU teeters on edge of a broader crisis", in: International Herald Tribune, 3.6.2005.

3 Vgl. den umfassenden Überblick von Jonas Brückner/Joachim Held/Andrea Stengel/Christian Völkel (2004): Der EU-Verfassungsprozeß. Bibliographie 2001-2004, Stiftung Wissenschaft und Politik SWP, August 2004, Berlin. 
Konstitutionalisierung ' in eine offene und auch öffentlich ausgetragene Debatte überführt hat.

\section{Der Verfassungskonvent - Chancen genutzt?}

Die erste große deutschsprachige Monografie zum europäischen Verfassungskonvent haben Peter Becker und Olaf Leiße vorgelegt. Die Autoren legen ihre Studie historisch an und ordnen die Arbeit des Konvents in die Reihe der Regierungskonferenzen seit Maastricht ein; dabei können sie zeigen, wie auf Grund der bisherigen Erfahrungen die Grenzen der traditionellen intergouvernementalen Methode offensichtlich wurde. Deshalb sahen sich, so stellen Becker und Leiße fest, die „Repräsentanten der Mitgliedstaaten zunehmend genötigt, außerhalb des eigentlichen europäischen Rahmens auf die Finalität der Europäischen Union einzugehen und nach neuen, kreativen Lösungen zur Auflösung des europäischen Reformstaus zu suchen“ “4. Da der im Juni 1999 eingesetzte Konvent zur Erarbeitung der Grundrechtecharta unter Vorsitz von Roman Herzog nach allgemeiner Überzeugung sehr erfolgreich gearbeitet hat, war die neue und kreative Methode gefunden. Der auf dem Gipfel von Laeken im Dezember 2001 aufgelegte Fragenkatalog bestimmte dann die Richtung der weiteren Debatte. In den Schlussfolgerungen von Laeken tauchte erstmalig expressis verbis in einem Gipfeldokument „Verfassung“ als mögliches Ziel der europäischen Politik auf - damit war ein Tabu gebrochen. Im dritten Teil ihrer Studie zeichnen die Autoren sehr detailliert und gut informiert die Struktur, die Arbeitsweise wie auch die Zusammensetzung des Konvents nach. Die einzelnen Plenarsitzungen in den drei vom Konventspräsidenten Giscard d'Estaing so bestimmten Arbeitsphasen sowie auch die Arbeit der „working groups“ werden jeweils knapp referiert und anhand der Dokumente sowie der begleitenden Medienberichterstattung und der Sekundärliteratur nachgezeich- net. In einem weiteren Kapitel (,Im Umfeld des Konvents") werden auch der Kontext, in dem der Konvent tagte (Stichworte: ,Irakkrise " und Spaltung in , altes' und ,neues Europa') aufgegriffen und der Frage nachgegangen, inwieweit solche externen Faktoren die Arbeit des Konvents beeinflusst haben. Die Autoren sehen gerade auch in der ,Immunität " des Konvents einen Vorteil gegenüber der klassischen Regierungskonferenz: „Während im Konvent die Arbeit fast unbehelligt vom Tagesgeschehen weitergeführt worden ist, war die Regierungskonferenz von bestimmten Konstellationen zwischen den Mitgliedstaaten und den persönlichen Beziehungen der Staatsund Regierungschefs hochgradig abhängig" ${ }^{* 5}$.

In ihrer abschließen Zusammenfassung kommen Becker und Leiße zu einem verhalten positiven Befund: Der Konvent habe zwar die ,großartige Chance, in einer freien und fairen Diskussionsatmosphäre die Zukunft Europas zu diskutieren“, genutzt und die darin liegenden Möglichkeiten ,,voll ausgeschöpft“. Eine „echte gesamteuropäische Debatte (...) über Chancen und Grenzen einer europäischen Demokratie, über Möglichkeiten des Zusammenlebens in Europa und das Ziel der Integration"6 fehle jedoch noch immer. Die Autoren haben mit ihrer umfassend und überzeugend angelegten Studie eine sehr gute Grundlage gelegt für alle diejenigen, die eine Antwort auf die Frage suchen, wie eine solche, noch ausstehende Debatte aussehen könnte. Im Anhang finden sich einige wenige Dokumente wie die „Erklärung für die Schlussakte zur Zukunft der Union" von Nizza sowie die Erklärung von Laeken und eine Zusammenstellung der Namen der Konventsvertreter.

\section{Der Konventsentwurf: Vorläufer-Ergebnisse - Fallstricke}

Auch die Sammelbände von Busek und Hummer sowie der von Mantl, Puntscher Riekmann und Schweitzer befassen sich schwer-

\footnotetext{
4 Becker/Leiße: Die Zukunft Europas, S. 25. 
punktmäßig mit dem Verfassungskonvent und seinem Ergebnis. Der Band Busek/Hummer versammelt acht Beiträge von Autoren, die als Diplomaten an den Beratungen der Regierungskonferenz mitgewirkt haben beziehungsweise von Wissenschaftlern, die sich seit vielen Jahren mit der Europäischen Union und ihren Reformen beschäftigen. Hervorgegangen ist der Band aus einer im Mai 2003 abgehaltenen Konferenz. Einen sehr guten Einstieg und weit in die Geschichte und Vorgeschichte der europäischen Integration zurückgreifenden Überblick bietet der Beitrag von Heinrich Schneider. Die Diskussion der ,Ursprünge und Vorläufer' der aktuelleren Verfassungsdiskussion erlaubt es Schneider, zwischen Traditionen und Paradigmawechseln der mal mehr oder weniger offen zu Tage tretenden Konstitutionalisierung der Europäischen Union klar zu unterscheiden. Eine Verfassung, die ihren Namen verdiene, sei ein Dokument, so Schneider, das nicht nur „formalen Erfordernissen“" entspreche, sondern eines, das ,dank seiner relativen Bestandsfestigkeit und dank der Loyalität aller politischer Kräfte normative Kraft" ${ }^{\text {"7 }}$ gewinne. Ähnlich grundlegend geht auch Waldemar Hummer in seinem Beitrag vor; er versieht den Zusammenhang zwischen „Konstitutionalisierung“ und „Verfassungsvertrag“ mit einem Fragezeichen. Was andere Autoren vielleicht vorschnell als gegeben ansehen, reflektiert er überzeugend unter anderem auf der Basis der in der Lehre und der Judikatur diskutierten Ansätze und kommt dann zu dem Ergebnis, dass die Begriffe ,Verfassung ' und ,Verfassungsvertrag ' mehr versprechen als sie einzulösen in der Lage sind - sie erwecken, so Hummer, „den Anspruch einer „Konstitutionalisierung" der (neuen) Union, ohne diesen aber einzulösen". 8 Andere Autoren untersu- chen die Zwischenergebnisse und die vom Konvent angedachten institutionellen Reformen (Elisabeth Tichy-Fisslberger), die Frage des Grundrechteschutzes in der Union (Christoph Grabenwarter), den Beitritt der Union zur Europäischen Menschenrechtskonvention (Wolfram Karl) sowie die Zusammenführung der Europäischen Union und der Europäischen Gemeinschaften (Walter Obwexer). Nach diesen eher an grundsätzlichen europarechtlichen Fragen ausgerichteten Beiträgen diskutieren Alfred Längle und Andreas Maurer die Frage, was passieren würde, wenn der Verfassungsvertrag einen oder mehrere „Ratifikationsunfälle“9 erleben würde, und welche Chancen dem Konventsergebnis in der nachfolgenden Regierungskonferenz einzuräumen sind. Längle plädiert für den nun eingetretenen Fall das Modell Dänemark (1992) und Irland (2001), wonach entsprechende Protokolle eine zweite Abstimmung über den Verfassungsvertrag erlauben würden; dies sei aber, so schränkt Längle zu Recht ein, nur möglich, wenn sich die Schwierigkeiten bei der Ratifikation ,nur auf materiellrechtliche Bestimmungen und nicht auch auf institutionelle beziehen, da bei Letzteren eine differenzierte Anwendung nicht möglich"10 sei. Maurer legt in seinem Beitrag die Grundprobleme des Modells Regierungskonferenz dar, die sich gerade auch bei den Verhandlungen von Nizza gezeigt haben - etwa die Tatsache, dass ein Mitgliedstaat wie Frankreich einerseits als Moderator die Verhandlungen voranbringen musste, gleichzeitig sich aber nicht von den eigenen nationalen Interessen befreien konnte oder wollte. Der durch viele Tabellen und breites empirisches Material angereicherte Beitrag Maurers diskutiert die Ergebnisse des Konvents und die Implikationen für das EUSystem in sehr überzeugender Weise. Im An-

7 Heinrich Schneider: Ursprünge und Vorläufer der gegenwärtigen „Verfassungsdiskussion“ in der Europäischen Union, in: Busek/Hummer: Der Europäische Konvent und sein Ergebnis, S. 31.

8 Waldemar Hummer: Die Europäische Union: Vom Tempel zur Verfassung - „Konstitutionalisierung“ der EU durch den „Verfassungsvertrag“?, in: Busek/Hummer: Der Europäische Konvent und sein Ergebnis, S. 55.

9 Alfred Längle: Übernahme der Ergebnisse des „EU-Zukunftskonvents“ in die Regierungskonferenz und Lösungsvarianten für allfällige „Ratifikationsunfälle“, in: Busek/Hummer: Der Europäische Konvent und sein Ergebnis, S. 136

10 Längle: Übernahme der Ergebnisse, in Busek/Hummer: Der Europäische Konvent und sein Ergebnis, S. 145. 
hang des Bandes Busek/Hummer ist der Entwurf des Verfassungsvertrags abgedruckt, wie er im Juli 2003 von Konvent verabschiedet wurde.

\section{Europarechtliche Aspekte}

Ähnliche Ziele wie der vorhergehende Band verfolgt die von Wolfgang Mantl, Sonja Puntscher Riekmann und Michael Schweitzer herausgegebene Publikation. Auch hier steht der Konvent im Mittelpunkt des Interesses und die daraus abgeleiteten primär europarechtlich orientierten Fragen und Probleme. Die Herausgeber bezeichnen den Konvent und seine Rolle bei der Vorbereitung der Regierungskonferenz und das damit einhergehende hohe Maß an Transparenz und Öffentlichkeit als „kleine Revolution“11. Die ersten beiden Beiträge beschäftigen sich mit der Frage, ob und inwiefern durch den Konvent die demokratische Legitimation der Union (Hubert Isak) beziehungsweise die Repräsentation verbessert werden könne (Johannes Pollak/ Peter Slominski). Die vorsichtig formulierte Hoffnung und These Isaks, dass der Konvent und seine relative Transparenz die „Akzep$\operatorname{tanz}(. .$.$) bei den BürgerInnen“ erhöhe und da-$ durch die „Legitimationsbasis der Union gestärkt" werde, muss auf Grund der Erfahrungen mit den gescheiterten Referenden in Frankreich und in den Niederlanden zwar als ,falsifiziert' gelten, trotzdem bleiben die grundsätzlichen Unterschiede und Vorteile gegenüber der traditionellen Methode der Regierungskonferenz, wie sie im Beitrag herausgearbeitet wurden, gültig. Durch einen systematischen Vergleich der repräsentativen ,Qualität‘ von Regierungskonferenzen und Konvent als Methode der Vertragsrevision beziehungsweise deren Vorbereitung kommen Pollak und Slominski zu dem Schluss, dass der Konvent das gegenwärtige Modell der Regierungskonferenz „langfristig ersetzen“12 solle. Die folgenden fünf Beiträge beschäftigen sich mit Fragen, wie sie auf der Ta- gesordnung des Konvents, aber auch seit vielen Jahren im europarechtlichen Forschungsdiskurs stehen: die Grundrechtecharta (Erich Vranes), die Rechtspersönlichkeit der Union (Walter Obwexer), die vertikale Kompetenzverteilung in der Europäischen Union (Thomas Eilmansberger) und die Vereinfachung der Rechtsakte (Bedanna Bapuly) sowie die Frage, inwieweit eine ,Wirtschaftsverfassung" für die Union sinnvoll und realisierbar wäre (Barbara Dutzler).

\section{Transnationale Demokratie im Werden?}

Eine andere, weiter gefasste Perspektive nimmt der von Ulrike Liebert, Josef Falke, Kathrin Packham und Daniel Allnoch herausgegebene Band ein. Die Arbeit des Verfassungskonvents nehmen die 20 Autorinnen und Autoren zum Anlass, der Frage nachzugehen, ob Europa sich ,,auf dem Weg zur transnationalen Demokratie“" (so der Titel des Bandes) befinde. Das „Verfassungsexperiment", das die Autoren untersuchen und analysieren, setzte mit der Krise der Regierungskonferenz von Nizza ein. In fünf Kapiteln werden der „Weg zur Verfassungsdebatte“, die ,Institutionelle Ordnung und neue Aufgaben“, ,Das demokratische Leben der Europäischen Union“, der „Konvent als Methode der Verfassungsgebung“ sowie im Kapitel „Ausblick und Zwischenbilanz" die Frage diskutiert, ob ein EU-weites Referendum als Bedingung sine qua non der Verfassungsgebung zu gelten habe (Lutz Hager) und wie weit die Europäische Union auf den Weg der transnationalen Demokratie gekommen ist (Ulrike Liebert). Ein ,Experiment' ist der Band auch in anderer Hinsicht: eine große Zahl der Autoren und Autorinnen sind Nachwuchswissenschaftler. Dieses Experiment ist sehr gelungen und sollte Nachahmer finden, da viele empirisch wie theoretisch überzeugende Arbeiten nicht den Weg an das akademische Tageslicht finden. Eine andere Besonderheit dieses von Liebert und anderen herausgegebenen Bandes

11 Mantl/Puntscher Riekmann/Schweitzer: Der Konvent, S. 8.

12 in: Mantl/Puntscher Riekmann/Schweitzer: Der Konvent, S. 55. 
stellt die sorgfältig edierte CD-ROM dar, die dem Band beigegeben ist. Darauf sind die vom Konventspräsidium erstellten Grundlagendokumente, die Mandate und Schlussberichte der Arbeitsgruppen, die Syntheseberichte sowie die in den Beiträgen genannten Dokumente festgehalten. Das Dokumentenverzeichnis am Ende des Bandes erleichtert das Auffinden der gesuchten Dokumente.

\section{Analysen zum Verfassungsvertrag}

Diesen ,Bonus“ bietet auch der von Werner Weidenfeld herausgegebene Band „Die Europäische Verfassung in der Analyse“. Auch hier ist dem Band eine CD-ROM beigelegt, auf der neben den Konventsdokumenten und Verfassungsentwürfen auch eine Chronologie sowie eine Dokumentation des Web-Projektes zur EU-Reform zu finden sind. ${ }^{13}$ In der bewährten Tradition der seit dem Vertrag von Maastricht in Gütersloh verlegten Analysebände nehmen die Autorinnen und Autoren auch den Verfassungsvertrag unter die Lupe und untersuchen den mit ihm unternommenen Schritt ,in Richtung postnationaler Staatlichkeit" ${ }^{\text {"14. Dabei }}$ werden zunächst der Verlauf des Prozesses und eine allgemeine Einordnung gegeben (Werner Weidenfeld, Annette Heuser, Volker Stör). Im zweiten und umfangreicheren Teil des Bandes werden die Reformergebnisse im Einzelnen diskutiert und kritisch reflektiert. In zum Teil knapp gefassten und doch sehr tief gehenden Analysen werden etwa die Werte und Ziele der Union (Almut Metz), die Grundrechtecharta (Kristina Notz), die „,neue Machtarchitektur“ (Janis A. Emmanouilidis), die Kompetenzordnung (Thomas Fischer), die offene Methode der Koordinierung (Almut Metz), die flexible Integration (Janis A. Emmanouilidis), die Themen Wirtschaft und Währung (Roman Maruhn), die soziale Dimension (Martin Brusis), die europäische „Innenpolitik“ (Franziska Hagedorn), die Entwicklung der Außen- und Si- cherheits- wie der Verteidigungspolitik (Franco Algieri und Thomas Bauer) sowie die „Nachbarschaftspolitik“ der Union untersucht (Iris Kempe). Am Ende des Bandes findet sich eine ausführliche und kommentierte Chronologie, die den „Verfassungsprozess“ seit Nizza nachzeichnet. Dieser Band sollte, wie die anderen auch, nicht nur innerhalb der wissenschaftlichen ,community' seine Leserschaft finden, sondern auch - denn das entspricht dem Selbstverständnis einer Stiftung und einem Institut, die sich der ,angewandten Politikforschung“ verschrieben haben - in der Öffentlichkeit, bei Multiplikatoren im Bereich der politischen Bildung wie auch in den Medien finden.

\section{Neuordnung des EU-Systems}

Der letzte hier zu besprechende Band aus der Reihe Europäische Schriften des IEP ist gleichzeitig der aktuellste: Er ist im Mai 2005 erschienen und versammelt insgesamt 28 Beiträge von Wissenschaftlern und auch EU-Politikern aus Brüssel und Berlin. Mathias Jopp und Saskia Matl haben mit dem von ihnen herausgegebenen Werk die „Konstitutionalisierung der EU“" (so der Untertitel des Bandes) sehr grundlegend untersucht. In den ersten Beiträgen wird von den Autoren eine ,integrationspolitische Gesamtbewertung" vorgenommen. Wolfgang Wessels legt in seinem Artikel den ,Suchscheinwerfer sowohl auf lange historische Grundlinien staatlicher Entwicklungen in den letzten fünf Jahrhunderten als auch auf die mittelfristige konstitutionelle Entwicklung des EU-Systems in den letzten fünfzig Jahren"15. Erst unter dieser longue $d u$ rée-Perspektive wird deutlich, dass - unabhängig von einem möglichen Scheitern des Verfassungsvertrags - die in der Europäischen Union vereinigten Staaten eine „neuartige Stufe einer langen Entwicklung des europäischen Staates"16 erreicht haben und schon längst Teil eines europäischen „fusionierten

13 Vgl. die Webseite www.eu-reform.de.

14 Weidenfeld: Europäische Verfassung in der Analyse, S. 13.

15 Wolfgang Wessels: Die institutionelle Architektur des Verfassungsvertrags: Ein Meilenstein in der Integrationskonstuktion, in: Jopp/Matl: Der Vertrag über eine Verfassung, S. 45-85, hier S. 46.

16 Wessels: Die institutionelle Architektur, in: Jopp/Matl: Der Vertrag über eine Verfassung, S. 85. 
Föderalstaates“ sind. Peter-Christian MüllerGraff unterstreicht in seiner Analyse die „grundsätzliche konzeptionelle Kontinuität des europäischen Gemeinwesens“, die durch den Verfassungsvertrag ,systemrational“ fortgebildet werde. ${ }^{17}$ Mit der für die rechts- und politikwissenschaftliche Forschung konstitutiven ,sui generis'-Formel beschäftigt sich Heinrich Schneider. Auch er unterstreicht die Kontinuitätslinien in der europäischen Verfassungsentwicklung und vertritt die These, dass mit dem vorliegenden Verfassungsvertrag - auch für den Fall, dass er die Ratifkationshürden doch noch überspringen sollte das europäische „Verfassungsprojekt noch nicht zu Ende“ sei. Die europäische „Verfassungspolitik", so Schneider, schließe auch „Bemühungen um die ,Bewusstseinsverfassung ' der Unionsbürger" ein. ${ }^{18}$ Auch Antje Wiener und Sonja Puntscher Riekmann untersuchen den Prozess der Konstitutionalisierung in dieser weiten Perspektive.

In einem zweiten Block untersuchen vier Autoren (Rudolf Hrbek, Peter Becker, Andreas Maurer und Christian Deubner) die Rolle der Regionen, die Kompetenzordnung sowie die Normenhierarchie und die Verstärkte Zusammenarbeit im Verfassungsvertrag. Dann werden die einzelnen Politikfelder und Themenbereiche wie Wirtschafts- und Währungsunion (Rolf Caesar/Wim Kösters sowie Christian Weise), der Raum der Freiheit, der Sicherheit und des Rechts (Friedemann Kaiser), die Bereiche Außen-, Sicherheits- und Verteidigungspolitik (Christoph Herrmann, Elfriede Regelsberger, Udo Diedrichs/Mathias Jopp) und auch das neue und angesichts der nun in die Kritik geratenen Erweiterungspolitik, interessante Politikfeld der Nachbarschaftspolitik (Barbara Lippert) behandelt. Des Weiteren kommen auch die Themen
,Partizipation“ und ,Legitimität" zur Sprache (Ulrike Liebert, Michael Piazolo, Oskar Niedermayer). Wie bei den anderen Bänden beschäftigen sich mehrere Autoren mit der Frage, was neu und innovativ zu nennen ist in der aktuellen Verfassungsdebatte. Die Beiträge von Daniel Göler und Hartmut Marhold, Brigid Laffan und Andreas Maurer diskutieren die „Verfassungsgebung als Prozess". Abgerundet wird der Band Jopp/Matl durch fünf Plädoyers von Politikern, die am Verfassungsprozess in verschiedenen Funktionen beteiligt waren (Hans Martin Bury, Elmar Brok, Jo Leinen, Peter Altmaier und Klaus Hänsch).

\section{Gegen die europapolitische Ratlosigkeit}

Zusammenfassend lässt sich festhalten, dass alle hier besprochenen Bände einen wichtigen Beitrag leisten für das Verständnis des europäischen ,Verfassungsprozesses'. Gerade in Zeiten der Unsicherheit und Ungewissheit darüber, ob und wie es weiter gehen soll, geben empirisch wie theoretisch fundierte Analysen eine stabile Basis für neue europapolitische Initiativen oder die kritische Reflexion des bisher Erreichten. Die im Umfeld der Ratifikationskrise im Frühsommer 2005 sichtbar gewordene Ratlosigkeit und die zahlreichen Appelle, jetzt endlich über den Sinn und Zweck der europäischen Integration nachzudenken, muten seltsam an. Wenn man die jüngste Geschichte der europäischen Integration kennt und das schon im Zusammenhang mit dem Konvent sichtbar gewordene Bemühen, solche grundlegenden Fragen zu diskutieren, sich in Erinnerung ruft, bleibt die Frage, weshalb dies in den letzten Jahren nicht ins allgemeine Bewusstsein getreten ist. Künftige Analysen werden diese und andere Fragen auf der Basis der hier vorgestellten Bände verfolgen und vertiefen können.

17 Peter-Christian Müller-Graff: Srukturmerkmale des neuen Verfasungsverrags für Europa im Entwicklungsgang des Primärrechts, in: Jopp/Matl: Der Vertrag über eine Verfassung, S. 87-107, hier S. 90.

18 Heinrich Schneider: Die neu verfasste Europäische Union: noch immer „das unbekannte Wesen“?, in: Jopp/ Matl: Der Vertrag über eine Verfassung, S. 109-132, hier S. 132. 